\title{
Information Theoretical Analysis of Synaptic Communication for Nanonetworks
}

\author{
Hamideh Ramezani ${ }^{1,2}$, Tooba Khan ${ }^{2}$ and Ozgur B. Akan ${ }^{1,2}$
}

\begin{abstract}
Communication among neurons is the highly evolved and efficient nanoscale communication paradigm, hence the most promising technique for biocompatible nanonetworks. This necessitates the understanding of neuro-spike communication from information theoretical perspective to reach a reference model for nanonetworks. This would also contribute towards developing ICT-based diagnostics techniques for neuro-degenerative diseases. Thus, in this paper, we focus on the fundamental building block of neuro-spike communication, i.e., signal transmission over a synapse, to evaluate its information transfer rate. We aim to analyze a realistic synaptic communication model, which for the first time, encompasses the variation in vesicle release probability with time, synaptic geometry and the re-uptake of neurotransmitters by pre-synaptic terminal. To achieve this objective, we formulate the mutual information between input and output of the synapse. Then, since this communication paradigm has memory, we evaluate the average mutual information over multiple transmissions to find its overall capacity. We derive a closed-form expression for the capacity of the synaptic communication as well as calculate the capacity-achieving input probability distribution. Finally, we find the effects of variation in different synaptic parameters on the information capacity and prove that the diffusion process does not decrease the information a neural response carries about the stimulus in real scenario.
\end{abstract}

Index Terms-Nanonetworks, molecular communication, neuro-spike communication, information capacity, synaptic transmission.

\section{INTRODUCTION}

Nanonetworks, foundation of the evolving field of Internet of Bio-nano Things (IoBNT), are formed to perform the complex tasks by expanding the capabilities of single Nanomachines [1]. They have a wide range of applications, such as intelligent drug delivery, health monitoring, development of nano-robots, nano-processors and nano-memories. Since molecular communication is found in biological systems, understanding of this phenomenon is anticipated to greatly facilitate the realization of bio-inspired nanoscale communication network [2], [3].

Information transmission within intra-body nervous system, known as neuro-spike communication, is one of the most investigated natural processes to understand molecular communication [4]-[6]. This communication is performed using two phenomena, i.e., propagation of electrochemical impulses within neurons and releasing neurotransmitters across adjacent

${ }^{1} \mathrm{H}$. Ramezani and O. B. Akan are with the Internet of Everything (IoE) Group, Electrical Engineering Division, Department of Engineering, University of Cambridge, UK

${ }^{2}$ H. Ramezani, T. Khan and O. B. Akan are with the Next-generation and Wireless Communications Laboratory (NWCL), Department of Electrical and Electronics Engineering, Koc University, Istanbul, Turkey

Emails: hr404@cam.ac.uk, tkhan15@ku.edu.tr, oba21@cam.ac.uk neurons. The electrochemical impulses are called action potential (AP) or spike that travel along the axon of a neuron. The transmitting neuron is termed as pre-synaptic neuron while the receiving neuron is called post-synaptic neuron as illustrated in Fig. 1. The axon of a pre-synaptic neuron branches out at the end to form connections with several post-synaptic neurons. The connection between these neurons, known as synapse, is a small gap forming a synaptic cleft between pre- and post-synaptic neurons. Arrival of a spike to the pre-synaptic terminal initiates the fusion of vesicles with the membrane. Then, the neurotransmitters stored inside the vesicle are released into the cleft, which then diffuse through the cleft and are captured by receptors present on post-synaptic terminal generating excitatory or inhibitory postsynaptic potential.

In nervous system, sensory information from outside world as well as signals from other organs are encoded into AP's, which are transmitted through a neuronal network to be processed by brain. Information theory provides a mean to estimate the transmission capacity of this complex communication network along with quantifying the reliability of the synaptic communication model [7] .

Neuro-spike communication has been studied from information theoretical perspective at different levels of complexities and for various network topologies [6], [8]-[10]. The capacity of neuro-spike communication is evaluated in [8], [11] using Hodgkin-Huxley $(\mathrm{HH})$ model through numerical simulations. In [9], the information transfer rate for single-input singleoutput (SISO) system has been calculated under signal detection and signal estimation paradigms. Moreover, the closedform expression for the capacity of SISO and multiple-input single-output (MISO) neuro-spike communication is derived in [6] using probabilistic model. This work is further extended in [12] to find the information rate in a SISO model with multiple synaptic terminals between two neurons. In [10], the upper bound of the capacity for a SISO synaptic communication is derived using Bernoulli distribution to model diffusion of neurotransmitters through the cleft, which ignores the synaptic geometry and the removal of residual neurotransmitters from the cleft. All the above cited studies have ignored the variability in the response due to (i) variations in vesicle release probability with time as explained in [13] and (ii) effect of synaptic geometry, diffusion of neurotransmitters and their clearance from the synaptic cleft, which would affect the mutual information, thus, capacity of the synaptic transmission. Recently, the variation in vesicle release probability is considered in [14], where it is shown that ignoring this variation causes overestimation of capacity of vesicle release 


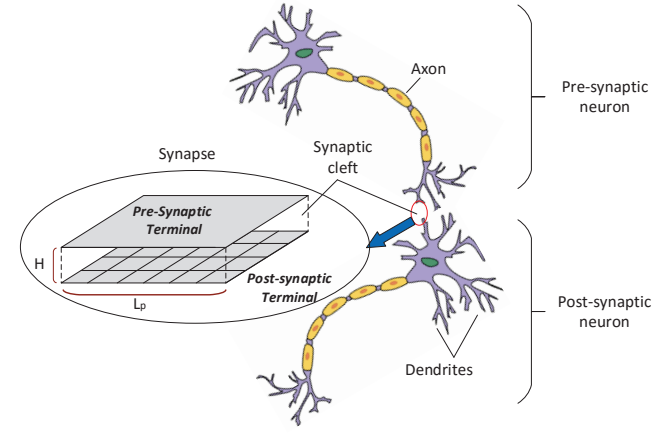

Fig. 1. Synaptic communication model.

process. Since, according to the data processing inequality, further processing does not increase the information, all of the above stated studies overestimated the capacity of the synaptic communication. Moreover, the capacity is only evaluated for the vesicle release process in [14], thus, there is a need to evaluate the capacity of complete synaptic model.

The main contribution of our paper is to derive analytical closed-form expressions for the information capacity of SISO synaptic transmission using realistic communication model for hippocampal pyramidal neurons. In our model, variation in vesicle release probability due to memory of neuron from previously released vesicles is taken into account. Moreover, the diffusion model that considers the synaptic geometry and clearance of neurotransmitters from the cleft between successive transmissions is used. Since the arrival of spike initiates the vesicle release process, the input of the system is considered as spike train. Moreover, the maximum number of bound receptors in response to each incoming spike is assumed as output since it contributes to generating the post-synaptic potential. We derive the information capacity and evaluate capacity-achieving input distribution for this SISO communication system for each incoming spike, i.e. for a single transmission. Then, to derive the overall capacity for multiple transmissions, we evaluate average mutual information using the steady-state release probability.

This whole study would serve as the basis for evaluating the capabilities of bio-inspired nanonetworks that utilize neurospike communication paradigm. Moreover, understanding of intra-body nervous nanonetworks from information theoretical perspective, would aid in designing ICT-inspired diagnostic and treatment techniques for neurodegenerative diseases that alter the typical ranges of the synaptic parameters. For instance, Alzheimer's disease (AD) reduces the available neurotransmitters [15], thus, decreasing the overall capacity of the synaptic transmission. Therefore, the calculation done in this paper would serve as the basis for the diagnosis of AD by comparing the capacity of healthy and diseased synapses.

The remainder of the paper is organized as follows. The synaptic communication model including vesicle release process, diffusion and receptor binding is presented in Section II. The overall capacity for multiple transmissions is derived in Section III, and numerical results are provided and analyzed

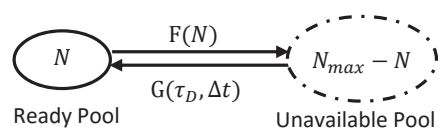

Fig. 2. Pool-based model for neurotransmitter release and replenishment.

in Section IV. Finally, the paper is concluded in Section V.

\section{Communication Theoretical Modeling of SYNAPTIC TRANSMISSION}

In this section, we present the communication model for three processes involved in synaptic transmission, i.e., vesicle release, diffusion and neurotransmitter-receptor binding. This communication model is later used to derive the maximum mutual information between input and output of the system.

\section{A. Vesicle Release Process}

Synaptic transmission is initiated with the arrival of spikes to the axonal terminal of the pre-synaptic neuron, which allows the influx of calcium ions by opening the voltage dependent calcium channels (VDCCs). After entering into the pre-synaptic terminal, calcium ions diffuse inside it, where they can bind to calcium buffers or sensors. Binding of enough calcium ions with a calcium sensor, which is located near the available vesicles for release, initiates the fusion of these vesicles with membrane. This causes the release of the content of vesicle, i.e., neurotransmitters, to the synaptic cleft [16].

In this paper, we consider existence of two distinct pools of vesicles in the pre-synaptic terminal as suggested in [13] and utilize the pool-based vesicle release and replenishment model shown in Fig. 2. In this model, the ready pool (RP) contains the vesicles ready for release, called readily releasable vesicles (RRVs), and the unavailable pool contains the released vesicles showing the capacity for replenishment of RP. In Fig. 2, the capacity of RP, i.e., the number of RRVs when no vesicle is released, is called $N_{\max } . F(N)$ is the vesicle release probability when $N, N \leq N_{\max }$, vesicles are available in RP. The mean recovery time for replenishment of one vesicle vacancy is $\tau_{D}$ and $G\left(\tau_{D}, \Delta t\right)$ is the probability of refilling one vesicle after $\Delta t$ seconds.

As it is mentioned in [13], the number of vesicles that are available to refill RP is much more than $N_{\max }$, hence, the replenishment of one released vesicle can be modeled by the first event of a Poisson process [17]. Thus, the probability of one vacancy replenishment is derived as

$$
G\left(\tau_{D}, \Delta t\right)=1-\exp \left(-\tau_{D}^{-1} \Delta t\right)
$$

and the number of recovered vesicles after $\Delta t$ seconds is derived based on Binomial distribution given by $B\left(N_{\max }-\right.$ $\left.N, G\left(\tau_{D}, \Delta t\right)\right)$.

Vesicles can release spontaneously or upon arrival of a spike. Hence, we consider both of these scenarios while deriving the vesicle release probability.

1) Evoked Release: After arrival of a spike to the presynaptic terminal until release of a vesicle, the release of every RRV is independent from others. However, after release of one 
vesicle, the others are temporarily prevented from release in hippocampal pyramidal neurons [13]. Hence, we utilize the uni-vesicular release model as given below.

The release probability for each of the existing vesicles in $\mathrm{RP}$ is governed by a Poisson process with rate $\lambda_{v}(t)$, which is a function of (i) opening of VDCCs and their distance from calcium sensors, (ii) binding of calcium ions to buffers, calcium sensors and pumps, and (iii) diffusion of calcium ions and buffers [18]. If a spike with duration equal to $\Delta t_{s}$ arrives at the pre-synaptic terminal at time $t_{0}$, the vesicle release happens in the time interval given by $\left[t_{0}, t_{0}+\Delta t_{s}\right]$. Thus, the fusion rate for each vesicle during a period of $\Delta t$ is defined as

$$
\alpha_{v}(\Delta t)=\int_{t_{0}}^{t_{0}+\Delta t} \lambda_{v}(t) d t
$$

where $\Delta t \leq \Delta t_{s}$. Then, as a result of using the univesicular release model, the probability of evoked release in the time interval $\left[t_{0}, t_{0}+\Delta t\right]$ is given as [6],

$P\{$ Release $\mid$ Spike arrival $\}= \begin{cases}1-\exp \left(-N \alpha_{v}(\Delta t)\right), & \Delta t \leq \Delta t_{s} \\ 1-\exp \left(-N \alpha_{v}\left(\Delta t_{s}\right)\right), & \Delta t>\Delta t_{s}\end{cases}$ where $N$ is the number of RRVs.

2) Spontaneous Release: The spontaneous vesicle release is independent from evoked release. Since the average waiting time for spontaneous release of each RRV is $480 \mathrm{~s}$ [19], the spontaneous release probability from a synapse with $N$ vesicles during $\Delta t$ seconds is derived as follows.

$$
P\{\text { Release } \mid \text { No spike }\}=1-\exp \left(-\frac{N \Delta t}{480}\right)
$$

\section{B. Diffusion Process}

After a vesicle release, neurotransmitters follow Brownian motion to diffuse across the synaptic cleft. We consider the synapse in the shape of a box with height $H$ as shown in Fig. 1. The top and bottom surfaces of the box correspond to the pre- and post-synaptic membranes, respectively, and are considered to be infinite in dimensions. This assumption is in accordance with the diffusion model provided in [20]. After vesicle is released from pre-synaptic terminal, neurotransmitters are diffused through the cleft and captured by post-synaptic receptors present on post-synaptic density (PSD). PSD occupies a limited square shaped region on the post-synaptic terminal with side length $L_{p}$ as shown in Fig. 1.

The concentration of neurotransmitters in three-dimension at any time $t$, denoted as $C(x, y, z, t)$, is random and its expected value can be calculated from Fick's equation given below,

$$
\frac{\partial C(x, y, z, t)}{\partial t}=D_{c} \nabla^{2} C(x, y, z, t),
$$

where $D_{c}$ is diffusion coefficient, $t \geq 0$ and $(x, y, z) \in \mathbb{R}^{2} \times$ $[0, H]$. While considering the reflections of neurotransmitters from pre- and post-synaptic membranes and in presence of presynaptic re-uptake of neurotransmitters, the solution of Fick's equation is given in [20]. This gives the expected concentration

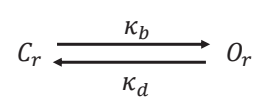

Fig. 3. Kinetic scheme of AMPA receptors.

of neurotransmitters in the cleft as follows,

$$
\begin{aligned}
C(x, y, z, t)= & \frac{T_{0}}{\left(\sqrt{4 \pi D_{c} t}\right)^{3}} e^{\frac{\left(-x^{2}-y^{2}\right)}{4 D_{c} t}} \\
& \left\{\sum_{k=-\infty}^{-1}\left(2-P_{u}\right)\left(1-P_{u}\right)^{-(k+1)} e^{\frac{-(z-(2 k+1) H)^{2}}{4 D_{c} t}}\right. \\
& \left.+\sum_{k=0}^{\infty}\left(2-P_{u}\right)\left(1-P_{u}\right)^{k} e^{\frac{-(z-(2 k+1) H)^{2}}{4 D_{c} t}}\right\},
\end{aligned}
$$

where $T_{0}$ is the number of neurotransmitters in a vesicle and $P_{u}$ [uptake/hit] is the uptake probability.

On the receiver side, the neurotransmitters hit the receptors and bind to them with some probability. According to [20], we consider that receptors are uniformly distributed on the PSD and the only neurotransmitters which are inside a small volume, i.e., $V_{e}$, around each receptor are likely to bind.

To calculate the expected concentration of neurotransmitters inside $V_{e}$, i.e., $C_{t}$, we assume that there is only one neurotransmitter in the cleft. Thus, according to [20], the probability of finding that neurotransmitter inside $V_{e}$ at time $t$ is given by

$$
P_{e}(t)=\iiint_{V_{e}} C(x, y, z, t) d x d y d z
$$

where $C$ is given by (5) with $T_{0}=1$.

According to [20], all particles move independently from each other. Thus, the number of neurotransmitters found inside $V_{e}$ at time $t$ can be represented by a binomial random variable, $T_{e}(t)$, having expected value

$$
E\left[T_{e}(t)\right]=T_{t}(t) P_{e}(t),
$$

where $T_{t}(t)$ is the expected total number of unbound neurotransmitters in the cleft at time t. Thus,

$$
C_{t} \approx \frac{E\left[T_{e}(t)\right]}{\left|V_{e}\right|}
$$

where $\left|V_{e}\right|$ is the size of the effective volume.

\section{Neurotransmitter-Receptor Binding}

PSD in hippocampal neurons contains two major kinds of receptors, i.e., AMPA ( $\alpha$-Amino-3-hydroxy-5-methyl-4isoxazolepropionic acid) and NMDA ( $N$-methyl-D-aspartate). However, we only consider AMPA receptors uniformly distributed in PSD since NMDA receptors contribute in synaptic plasticity, which is not addressed in this paper. Binding of neurotransmitters to AMPA receptors increases membrane potential of post-synaptic neuron, i.e., generates excitatory post-synaptic potential (EPSP).

To derive the probability of neurotransmitter binding to an AMPA receptor, we utilize the simplified kinetic model demonstrated in Fig. 3. As it is shown in [21], this simplified 


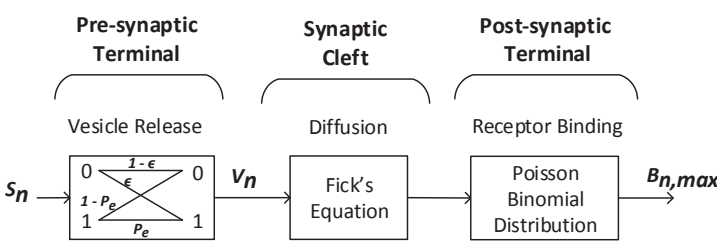

Fig. 4. Block diagram of the synaptic communication.

model provides fairly good approximations for the time course and the dynamic behavior of synaptic currents compared to the complex multi-state kinetic models.

When receptor $r, r \in\left[1, R_{0}\right]$, is in the closed state, i.e., $C_{r}$, it is available for binding to a neurotransmitter. After binding, the receptor moves to the open state, i.e., $O_{r}$, and allows the influx of ions that changes the membrane potential of post-synaptic terminal. The probability of being in each of these states changes with time depending on the expected concentration of neurotransmitters near receptor, i.e., $C_{t}$, as given in the following equations,

$$
\begin{gathered}
\frac{d C_{r}}{d t}=-\kappa_{b} C_{r} C_{t}+\kappa_{d} O_{r}, \\
C_{r}+O_{r}=1,
\end{gathered}
$$

where $\kappa_{b}$ and $\kappa_{d}$ are binding and dissociation rates for AMPA receptors, respectively. $O_{r}$ and $C_{r}$ also represent the opening and closing probabilities of AMPA receptors, respectively, that depend on both time and the position of the receptors.

\section{CAPACITY ANALYSIS OF SyNAPtiC COMMUNICATION}

The extensive analysis of synaptic transmission from information theoretical perspective is required to lay down the foundation of bio-inspired nanonetworks. Thus, in this section, we aim to derive the closed-form expression of the capacity of the synaptic transmission that depends on the availability of readily releasable vesicles, which changes with time and depends on the previous vesicle releases. Therefore, we discretize time into windows of equal durations, i.e., $\Delta t$, and select $\Delta t$ sufficiently small so that at most one spike can exist at each window [6]. Note that by considering the width of spike equal to $\Delta t_{s}, \Delta t$ must be selected greater than or equal to $\Delta t_{s}$ to have complete spike duration in one window.

In each time step, we can maximize mutual information over all possible spiking probabilities to estimate capacity of the system. However, since the number of RRVs changes over time, the vesicle release probability also changes. Hence, according to the definition, mutual information would also vary with time. Thus, we need to find average mutual information from 1 st to $n$th time step and derive the spiking probability that maximizes it to reach the overall capacity until time step $n$. Finally, we extend this calculation to find the capacity of synaptic transmission when $n$ approaches to infinity.

\section{A. Mutual Information in Each Time Step}

The input and output of the synaptic communication model shown in Fig. 4 are $S_{n}$ and $B_{n, \max }$, respectively, in $n$th time slot. Here, $S_{n}$ depicts the existence of spike at input and $B_{n, \max }$ shows the maximum number of bound receptors at the postsynaptic terminal as a result of vesicle release. Hence, the mutual information of the system at each time step can be defined as follows,

$$
I\left(S_{n}, B_{n, \text { max }}\right)=\mathcal{H}\left(B_{n, \text { max }}\right)-\mathcal{H}\left(B_{n, \text { max }} \mid S_{n}\right),
$$

where the entropies in (10) follow the general formula given as $\mathcal{H}\left(\mathcal{X}_{n}\right)=-\sum_{i} P\left\{X_{n}=i\right\} \log _{2} P\left\{X_{n}=i\right\}$.

By conditioning over the spike arrival, we can write the probability $P\left\{B_{n, \max }=i\right\}$ as given below,

$$
P\left\{B_{n, \max }=i\right\}=\sum_{j=0}^{1} P\left\{B_{n, \max }=i \mid S_{n}=j\right\} P\left\{S_{n}=j\right\},
$$

where $i \in\left[0, R_{0}\right]$ and $R_{0}$ is the total number of receptors on the post-synaptic terminal. Same as [6], [9], we model the spike arrival with a Poisson process. Hence, assuming the rate of this process as $\lambda$, the probability of spike arrival at $n$th time slot can be calculated as $P\left\{S_{n}=1\right\}=1-\exp (-\lambda \Delta t) \triangleq p$.

To derive the probability $P\left\{B_{n, \max }=i \mid S_{n}=j\right\}$, we can condition over the vesicle release process as follows.

$$
\begin{aligned}
& P\left\{B_{n, \text { max }}=i \mid S_{n}=j\right\}= \\
& \sum_{k=0}^{1} P\left\{B_{n, \text { max }}=i \mid V_{n}=k\right\} P\left\{V_{n}=k \mid S_{n}=j\right\},
\end{aligned}
$$

where $V_{n}$ indicates the release of vesicle in $n$th window. Since the neurotransmitters are removed from synaptic cleft between successive vesicle releases through re-uptake and diffusion [20], the synaptic noise due to residual neurotransmitters is not significant in healthy neurons and we consider $P\left\{B_{n, \max }=\right.$ $\left.0 \mid V_{n}=0\right\}=1$.

To find the probability $P\left\{B_{n, \max }=i \mid V_{n}=1\right\}$, we define the opening probability of $r$ th receptor as $O_{r}(t)$, where $r \in\left[1, R_{0}\right]$ and $0 \leq t \leq \Delta t$. As shown in [20], a smaller time step called $\Delta \tau<<\Delta t$ can be used to calculate $O_{r}(t)$ in each $\Delta t$. We are interested in the maximum number of bound receptors, $B_{n, \max }$, since it contributes to the peak of EPSP. After binding to a neurotransmitter, the receptors stay in open state until EPSP reaches its maximum value. Hence, utilizing the maximum opening probability for $r$ th receptor during $n$th time step, i.e., $O_{r, n}=\max _{0 \leq t \leq \Delta t}\left(O_{r}(t)\right)$, we can calculate $B_{n, \max }$ by defining the variable $x_{r, n}$ as follows.

$$
x_{r, n}=\left\{\begin{array}{ll}
0, & 1-O_{r, n} \\
1, & O_{r, n}
\end{array} .\right.
$$

The $\sum_{r=1}^{R_{0}} x_{r, n}$ shows the maximum number of open receptors during time step $\Delta t$. Hence, the probability of having $i$ open receptors upon vesicle release, i.e., $P\left\{B_{n, \max }=i \mid V_{n}=1\right\}$, is equal to $P\left\{\sum_{r=1}^{R_{0}} x_{r, n}=i\right\}$, which can be modeled by Poisson Binomial distribution with mean, $\mu_{n}$, and variance, $\sigma_{n}^{2}$, as shown in [22],

$$
\mu_{n}=\sum_{r=1}^{R_{0}} O_{r, n}
$$




$$
\sigma_{n}^{2}=\sum_{r=1}^{R_{0}} O_{r, n}\left(1-O_{r, n}\right)
$$

Thus, the probability $P\left\{B_{n, \max }=i \mid V_{n}=1\right\}$ is calculated as follows,

$$
P\left\{B_{n, \max }=i \mid V_{n}=1\right\}=\frac{1}{R_{0}+1} \sum_{l=0}^{R_{0}} E^{-l i} \prod_{r=1}^{R_{0}}\left[1+\left(E^{l}-1\right) O_{r, n}\right],
$$

where $E=\exp \left(\frac{2 \sqrt{-1} \pi}{R_{0}+1}\right)$

Next step is deriving the conditional vesicle release probability given in (12), i.e., $P\left\{V_{n}=k \mid S_{n}=j\right\}$. As it is shown in Fig. 4, a binary channel can be used to model the vesicle release process at each time step. According to (2) and (3) and the pool-based vesicle release and replenishment model depicted in Fig. 2, the probability of release in $n$th time slot when RP has $N$ vesicles is expressed as follows,

$$
\begin{gathered}
P\left\{V_{n}=1 \mid N_{n}=N, S_{n}=1\right\}=1-\exp \left(-N \alpha_{v}\left(\Delta t_{s}\right)\right) \triangleq P_{e}, \\
P\left\{V_{n}=1 \mid N_{n}=N, S_{n}=0\right\}=1-\exp \left(-\frac{N \Delta t}{480}\right) \triangleq \epsilon,
\end{gathered}
$$

where (17) shows the probability of evoked release, (18) depicts the spontaneous release probability and $N_{n}$ indicates the number of available vesicles in RP at the beginning of $n$th time slot. Then, the conditional vesicle release probability can be written as follows,

$$
\begin{aligned}
& P\left\{V_{n}=k \mid S_{n}=j\right\}= \\
& \quad \sum_{N=0}^{N_{\max }} P\left\{V_{n}=k \mid N_{n}=N, S_{n}=j\right\} P\left\{N_{n}=N\right\} .
\end{aligned}
$$

In [14], an array named $P_{n}$ is defined with dimension $1 \times$ $\left(N_{\max }+1\right)$ having $i$ th element equal to $P\left\{N_{n}=i-1\right\}$. Then, a recurrence relation is derived for $P_{n}$ as follows,

$$
P_{n}=P_{n-1} D R .
$$

Here, $D$ and $R$ are $\left(N_{\max }+1\right) \times\left(N_{\max }+1\right)$ matrices indicating the impact of pool depletion and replenishment on the probability of having a given number of RRVs, respectively. To derive the probability of having different number of RRVs in time slot $n$, (19) is simplified to $P_{n}=P_{1}(D R)^{n-1}$, where $P_{1}$ is equal to $[0,0, \ldots, 0,1]$ indicating that RP is full, i.e., $N=N_{\max }$, before the arrival of spikes.

The $(i, j)$ th element of $D$, i.e., $D_{i j}$, represents the probability of transition from having $N_{n-1}=i-1$ to $N_{n}=j-1$ as a result of vesicle release. Since we are using the univesicular release model, at most one vesicle can be released from the pool. Hence, the nonzero elements of matrix $D$ occur only where $j \in\{i, i-1\}$ and can be derived from vesicle release probability shown in Fig. $2, F_{n}($.$) , as D_{i i}=1-F_{n}(i-1)$ and $D_{i(i-1)}=$ $F_{n}(i-1)$. Using the spiking probability, $p$, and the probabilities given in (2) and (3), the vesicle release probability in $n$th slot when RP has $i$ vesicles, i.e., $F_{n}(i)$, involving both spontaneous and evoked releases, is derived as follows,

$$
F_{n}(i)=1-\left[\exp \left(-i \alpha_{v}\left(\Delta t_{s}\right)\right) p+\exp \left(-\frac{i \Delta t}{480}\right)(1-p)\right] .
$$

The $(i, j)$ th element of $R$, i.e., $R_{i j}$, represents the probability of transition from having $N_{n-1}=i-1$ to $N_{n}=j-1$ as a result of vesicle replenishment process. Since the number of refilled vesicles when the RP has $i$ RRVs can be derived from Binomial distribution given by $B\left(N_{\max }-i, G\left(\tau_{D}, \Delta t\right)\right)$ as explained in Section II-A, the matrix $R$ is an upper triangular matrix whose $(i, j)$ th element for $\forall j \geq i$ is defined as follows,

$$
R_{i j}=\left(\begin{array}{c}
N_{\max }-(i-1) \\
j-i
\end{array}\right) G\left(\tau_{D}, \Delta t\right)^{j-i}\left(1-G\left(\tau_{D}, \Delta t\right)\right)^{N_{\max }-(j-1)} .
$$

\section{B. Information Capacity of synaptic transmission}

We define the maximum mutual information achieved during first $n$ time slots as $C_{n}=\max _{p} \sum_{l=1}^{n} \frac{I\left(S_{l} ; B_{l, \max }\right)}{n}$. Then, the overall capacity of synaptic communication is defined as $C=\lim _{n \rightarrow \infty} C_{n}$, for which we evaluate the convergence of $I\left(S_{n} ; B_{n, \max }\right)$. Based on the recurrent equation given by (19), the number of vesicles in RP can be modeled by a finitestate Markov chain. Moreover, the steady state probability of each state in this Markov chain, i.e., $\pi=\left[\pi_{0}, \pi_{1}, \ldots, \pi_{N_{\max }}\right]$, can be derived by solving the linear equations $\pi=\pi D R$ and $\sum_{i=0}^{N_{\max }} \pi_{i}=1$. Then, to find $\lim _{n \rightarrow \infty} I\left(S_{n} ; B_{n, \max }\right)$, we calculate the limit of (12) when $n$ tends to infinity as follows,

$$
\begin{aligned}
& \lim _{n \rightarrow \infty} P\left\{B_{n, \text { max }}=i \mid S_{n}=j\right\}= \\
& \sum_{k=0}^{1} P\left\{B_{n, \text { max }}=i \mid V_{n}=k\right\} \sum_{N=0}^{N_{\max }} P\left\{V_{n}=k \mid S_{n}=j, N_{n}=N\right\} \pi_{N} .
\end{aligned}
$$

Now, the limit of (11) as $n$ tends to infinity is found as

$$
\begin{aligned}
& \lim _{n \rightarrow \infty} P\left\{B_{n, \text { max }}=i\right\}= \\
& \sum_{j=0}^{1} P\left\{S_{n}=j\right\} \lim _{n \rightarrow \infty} P\left\{B_{n, \max }=i \mid S_{n}=j\right\} .
\end{aligned}
$$

Therefore, the mutual information, $I\left(S_{n} ; V_{n}\right)$, converges as given below.

$$
\begin{aligned}
\lim _{n \rightarrow \infty} I\left(S_{n} ; B_{n, \text { max }}\right) & =\mathcal{H}\left(\lim _{n \rightarrow \infty} B_{n, \text { max }}\right)-\mathcal{H}\left(\lim _{n \rightarrow \infty} B_{n, \text { max }} \mid S_{n}\right) \\
& \triangleq I_{\infty},
\end{aligned}
$$

where the entropies in the above equation follow the general formula given as

$$
\mathcal{H}\left(\lim _{n \rightarrow \infty} \mathcal{X}_{n}\right)=-\sum_{i} \lim _{n \rightarrow \infty} P\left\{X_{n}=i\right\} \log _{2}\left(\lim _{n \rightarrow \infty} P\left\{X_{n}=i\right\}\right) .
$$

Considering the binary channel of vesicle release block in Fig. 4, since the crossover probability when there is no spike at input, $\epsilon$, is very small in real scenario, the mutual information at the output of this block decreases if $P_{e}$ reduces. Since the number of RRVs decreases with time as a result of pool depletion; according to (17), the evoked release probability, $P_{e}$, thus, $I\left(S_{n} ; V_{n}\right)$, also reduces with time. Moreover, according to data processing inequality, further processing does not increase the information. Hence, $I\left(S_{n} ; B_{n, \max }\right)$ also decreases with time. Furthermore, according to (21), I( $\left.S_{n} ; B_{n, \max }\right)$ converges to $I_{\infty}$, thus, its average over time also converges to $I_{\infty}$, i.e.,

$$
\lim _{n \rightarrow \infty} \sum_{l=1}^{n} \frac{I\left(S_{l} ; B_{l, \max }\right)}{n}=I_{\infty},
$$


TABLE I

SiMULATION PARAMETERS

\begin{tabular}{|l|l|l|}
\hline Parameters & Symbols & Values \\
\hline Synaptic cleft height & $H$ & $20 \mathrm{~nm} \mathrm{[23]}$ \\
\hline Diffusion coefficient & $D_{c}$ & $0.33 \mu \mathrm{m}^{2} / \mathrm{ms}[24]$ \\
\hline Side length of PSD & $L_{p}$ & $0.4 \mu \mathrm{m} \mathrm{[20]}$ \\
\hline Number of receptors & $R_{0}$ & $441[25]$ \\
\hline Pre-synaptic Re-uptake & $P_{u}$ & $10 \%[20]$ \\
\hline Binding Rate of AMPA & $\kappa_{b}$ & $78 \times 10^{6} \frac{1}{\mathrm{Ms}}[26]$ \\
\hline Dissociation Rate of AMPA & $\kappa_{d}$ & $750 \mathrm{~s}^{-1}[26]$ \\
\hline Effective Volume & $V_{e}$ & $1 \times 1 \times 0.5 \mathrm{~nm}^{3}$ \\
\hline Simulation time step & $\Delta \tau$ & $3.85 \mathrm{~ns}[20]$ \\
\hline Spike width & $\Delta t_{s}$ & $4 \mathrm{~ms}[16]$ \\
\hline Discretization time step & $\Delta t$ & $\Delta t_{s}[14]$ \\
\hline Average fusion rate & $\alpha_{v}\left(\Delta t_{s}\right)$ & $0.06 \sqrt{N}[27]$ \\
\hline Mean vacancy recovery time & $\tau_{D}$ & $\frac{0.6}{N_{\max }}[17]$ \\
\hline
\end{tabular}

and the capacity of synaptic communication can be calculated as follows,

$$
C=\max _{p} I_{\infty}
$$

\section{Evaluation}

In this section, we intend to evaluate the capacity of the synaptic communication and find how it is affected by varying different synaptic parameters. Since the output of the system, i.e., the maximum number of bound receptors, depends on spiking probability, $p$, capacity of RP, $N_{\max }$, and number of neurotransmitters in a vesicle, $T_{0}$, we first evaluate the impact of these parameters on the mutual information between input and output. The values of all simulation parameters are selected from the experimental studies as shown in Table I. Furthermore, we highlight how the mutual information changes over the multiple synaptic transmissions. Thus, we calculate the average mutual information over $n$ transmissions and maximize it to find the capacity until $n$th time slot. Finally, with the use of steady-state probability of number of RRVs, we find the overall mutual information and capacity along with the capacity-achieving input probability distribution.

\section{A. Mutual Information in Each Time Step}

In this section, we calculate the mutual information in each time step for different values of synaptic parameters, i.e., $N_{\max }$ and $T_{0}$, and spiking probability, $p$, and discuss the effects of changing these parameters on information transfer rate. Since the vesicle release process is the only part of the system that has memory, i.e., the number of RRVs changes after each transmission, we analyze the impact of variation of number of RRVs with time on mutual information. Among the aforementioned parameters, $T_{0}$ is not affecting the number of RRVs, hence the average number of available vesicles for release is plotted for different values of $N_{\text {max }}$ and $p$ in Fig. 5.

The pool-based vesicle release and refill model shown in Fig. 2 is used to derive the statistics of number of RRVs. As it is shown in Fig. 5, the number of RRVs reduces with time, thus, we can conclude that the release rate of vesicles

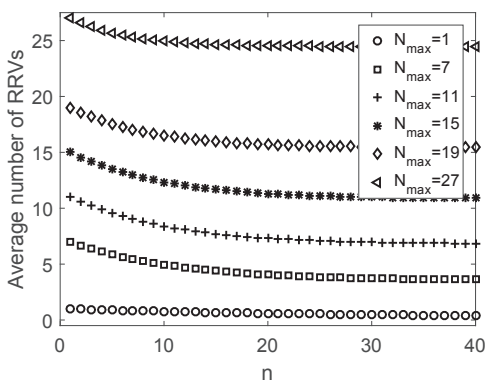

(a) $T_{0}=500, p=0.5$

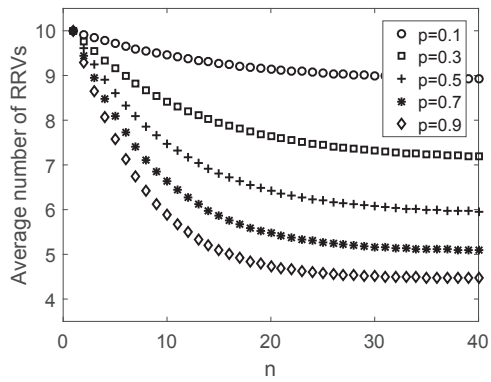

(b) $N_{\max }=10, T_{0}=500$

Fig. 5. Average number of available vesicle for release for different (a) capacity of ready pool, $N_{\max }$ and (b) spiking probabilities, $p$.

is faster than their refill rate, i.e., the neuron does not have enough time between multiple transmissions to refill all of the released vesicles. It can also be observed in Fig. 5(a) that increasing $N_{\max }$ increases the average number of vesicles available in each time slot. This is due to two reasons, i.e., higher value of $N_{\max }$ results in (i) having more number of RRVs in the beginning of simulation, and (ii) faster vacancy replenishment according to the definition of $\tau_{D}$ given in Table I. Moreover, according to Fig. 5(b), increasing the spiking probability, $p$, while keeping $N_{\max }$ fixed, decreases the average number of RRVs. Since the evoked release probability, $P_{e}$, is higher than spontaneous release probability, $\epsilon$, increasing spiking probability, $p$, causes the release of more number of vesicles in multiple transmissions. Moreover, the refill rate is constant, thus, the average number of RRVs decreases with time by increasing spiking probability, $p$.

The mutual information between input and output of the system is shown in Fig. 6 for different values of $N_{\max }, T_{0}$ and $p$. It is observed that the mutual information reduces over time. The reason of this reduction can be explained considering the binary channel of vesicle release block in Fig. 4. Since the crossover probability when there is no spike at input, $\epsilon$, is very small in real scenario, the mutual information at the output of this block would be maximum if $\left(1-P_{e}\right)$ tends to zero, i.e., as the evoked release probability, $P_{e}$, increases. On the other hand, the diffusion and binding processes cannot increase the information about input according to the data processing inequality. Thus, the overall mutual information of the system is directly related to the evoked release probability. According to (17), this release probability decreases with reduction in the number of RRVs and the average number of RRVs reduces with time as shown in Fig. 5. Therefore, mutual information 


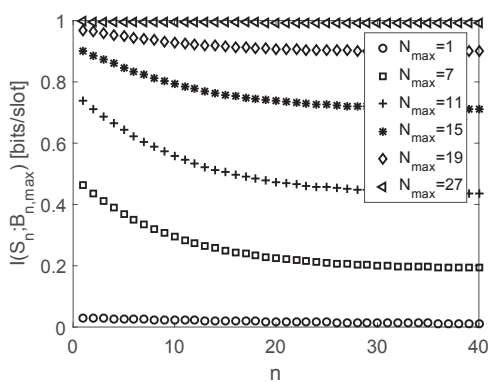

(a) $T_{0}=500, p=0.5$

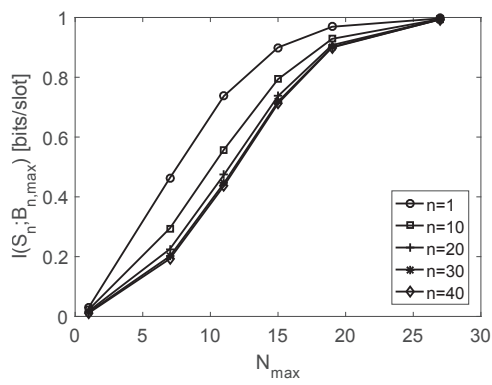

(d) $T_{0}=500, p=0.5$

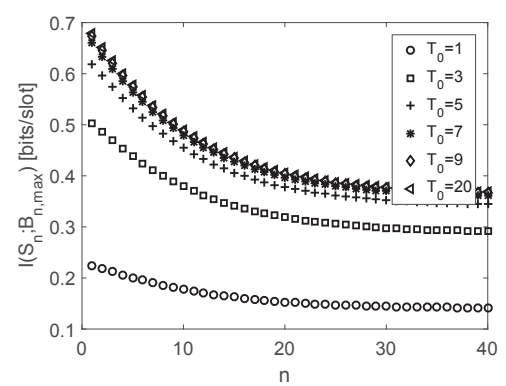

(b) $N_{\max }=10, p=0.5$

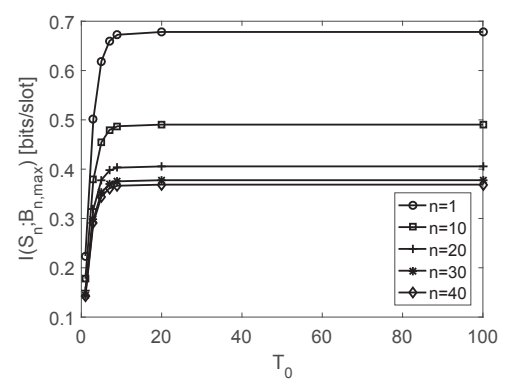

(e) $N_{\max }=10, p=0.5$

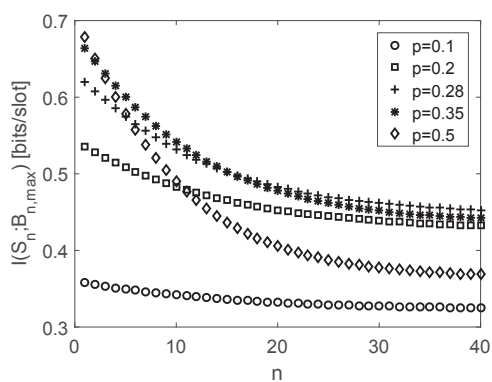

(c) $N_{\max }=10, T_{0}=500$

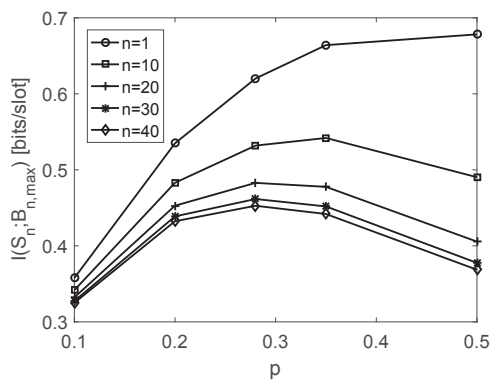

(f) $N_{\max }=10, T_{0}=500$

Fig. 6. Mutual information between spiking and maximum number of bound receptors for different (a,d) capacity of ready pools, $N_{m a x}$, (b,e) number of neurotransmitters in a vesicle, $T_{0}$, and (c,f) spiking probabilities, $p$.

also reduces over time as shown in Fig. 6. Moreover, since the number of RRVs, $N_{n}$, eventually reaches its steady state value according to the Markov chain model indicated in Section III-B, mutual information also saturates to a constant value.

In Fig. 6(a), the mutual information is plotted against time for different values of $N_{\max }$. It can be observed that the reduction rate of mutual information decreases with the increase in the value of $N_{\max }$. This is due the fact that high values of $N_{\max }$ leads to higher number of RRVs, $N_{n}$, in each time slot as shown in Fig. 5(a), which saturates the evoked release probability, $P_{e}$, according to (17). Therefore, the rate of change of mutual information tends to zero for higher values of $N_{\max }$ as shown in Fig. 6(a) and Fig. 6(d). Furthermore, for very low values of $N_{\text {max }}$, the signal transmission happens rarely, thus, mutual information is close to zero for all time.

It can also be observed in Fig. 6(a) and Fig. 6(d) that in each time slot we have higher value of mutual information as the value of $N_{\max }$ increases. This is due to having more number of RRVs, thus, higher evoked release probability, as a result of increasing $N_{\text {max }}$.

Next, we evaluate the effect of changing the number of neurotransmitters in a vesicle, $T_{0}$, on mutual information. We select $N_{\max }$ as 10, which is reported in [28] for average capacity of RP in hippocampal pyramidal neurons. As can be observed in Fig. 6(b) and Fig. 6(e), the output has more information about input if there is a higher number of neurotransmitters in a vesicle. Moreover, the mutual information attains saturation after certain value of $T_{0}$ as a result of having enough bound receptors to detect the signal transmission in case of spike arrival. Furthermore, for very low values of $T_{0}$, the neurotransmitters are lost in the cleft or uptaken by the presynaptic terminal before reaching the post-synaptic receptors, thus, resulting in lower value of mutual information.

The change in mutual information of synaptic transmission with respect to spiking probability is shown in Fig. 6(c) and Fig. 6(f). The mutual information decreases with higher rate with increasing spiking probability as shown in Fig. 6(c). This is due to more reduction in the number of RRVs by increasing spiking probability as shown in Fig. 5(b). Moreover, since the number of RRVs, $N_{n}$, changes with time, the spiking probability that maximizes mutual information also changes in each time slot. This change is apparent in Fig. 6(f) as the peak of mutual information is moving towards left.

The mutual information between spiking and output of vesicle release process is reported in [14]. By comparing the achievable mutual information for the overall synaptic communication shown in Fig. 6 and the results of [14], we can see that the diffusion and binding processes do not decrease the mutual information after certain value of $T_{0}$. Thus, we can conclude that if the value of $T_{0}$ is very high, which is the case in real scenario [29], enough amount of the released vesicle are always received by the post-synaptic neuron, which shows the reliability of the synaptic system.

\section{B. Average Mutual Information and Capacity for $n$ Synaptic Transmissions}

In the previous section, we observed that the mutual information changes with time, hence we need to find average mutual information over several transmissions to evaluate the capacity of synaptic communication. The mutual information averaged over $n$ consecutive time slots, always starting from the first time slot, is shown in Fig. 7. It is apparent in the figure that average mutual information reduces as the system use increases, which is a result of the depletion of RP. Moreover, 


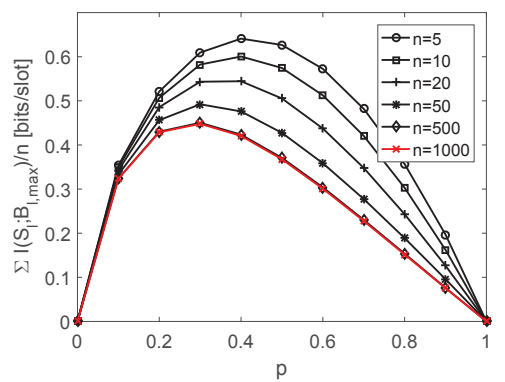

Fig. 7. Average mutual information for $N_{\max }=10$ and $T_{0}=500$.

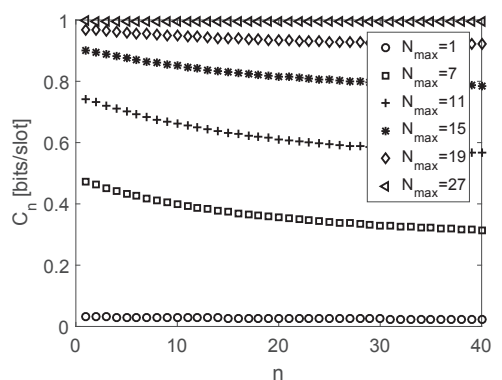

(a) $T_{0}=500$

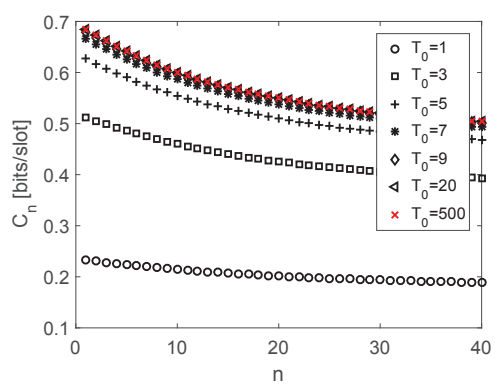

(b) $N_{\max }=10$

Fig. 8. Maximum mutual information from 1 st to $n$th slot for different values for (a) capacity of RP, $N_{\max }$, and (b) number of neurotransmitters in a vesicle, $T_{0}$.

the spiking probability that maximizes the average mutual information is also decreasing with time as indicated by the peak of the curve that moves towards left.

The average mutual information is maximized over spiking probability, $p$, to calculate the capacity over multiple transmissions, for different values of $N_{\max }$ and $T_{0}$ as shown in Fig. 8 . The capacity reduces with time as a result of reduction in mutual information over multiple transmissions as observed in Fig. 6. Moreover, increase in both $N_{\max }$ and $T_{0}$ increases the capacity as a result of higher vesicle release probability and increased chances of neurotransmitters to reach the receptors, respectively. Furthermore, as we have seen in Fig. 6(e), the mutual information in each time step saturates with increasing $T_{0}$. Hence, it can be observed in Fig. 8(b) that the capacity of the system over multiple uses also saturates after a specific value of $T_{0}$.

\section{Mutual Information and Capacity at Steady-State}

To evaluate the capacity of synaptic communication, we calculated the steady state probabilities of having different

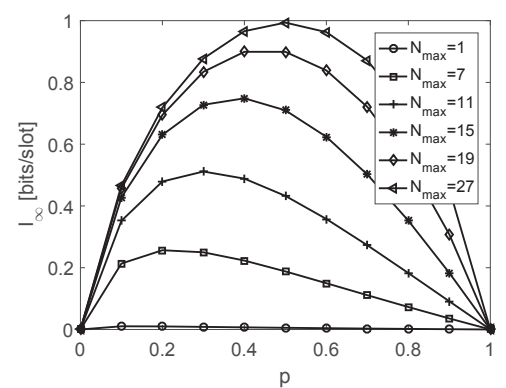

(a) $\lim _{n \rightarrow \infty} \sum_{l=1}^{n} \frac{I\left(S_{l} ; V_{l}\right)}{n}=I_{\infty}$

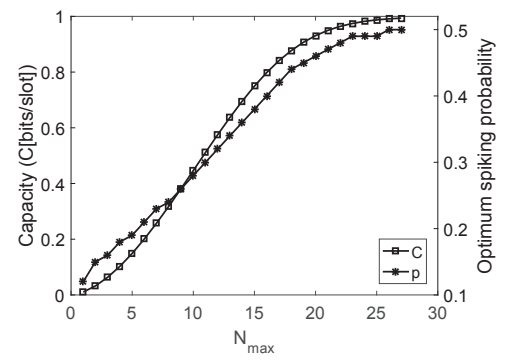

(b) Channel capacity and optimum spiking probability

Fig. 9. Average mutual information of the system, system capacity and capacity-achieving spiking probability in steady state for $T_{0}=500$ and different values of $N_{\max }$.

number of RRVs and use them to derive the mutual information between input and output of the system as $n$ tends to infinity, $I_{\infty}$. This also corresponds to the average mutual information evaluated over infinite consecutive time slots as given by (22).

The $I_{\infty}$ is plotted against spiking probability for different values of $N_{\max }$ and $T_{0}$ in Fig. 9(a) and Fig. 10(a), respectively. Similar to the average mutual information, we can observe that $I_{\infty}$ also increases with $N_{\max }$ and $T_{0}$. Moreover, $I_{\infty}$ is maximized over spiking probability, $p$, to calculate the capacity for different values of $N_{\max }$ and $T_{0}$ as shown in Fig. 9(b) and Fig. 10(b), respectively. Since changing the $N_{\max }$ affects the number of RRVs, which in turn affects the steady state probabilities of having different number of RRVs, the capacity-achieving spiking probability also changes as shown in Fig. 9. However, as it can be seen in Fig. 10, changing $T_{0}$ does not change capacity-achieving spiking probability since it is not affecting the availability of vesicles.

It can also be observed from Fig. 9(b) and Fig. 10(b) that capacity improves with increasing $N_{\max }$ and $T_{0}$, respectively. This is a result of dependence of $I_{\infty}$ on these factors as explained earlier. As it can be observed in Fig. 10(b), the capacity reaches the maximum value for $T_{0}$ around 10 . However, more number of bound receptors is needed for spike generation, thus, there are more neurotransmitters in a vesicle in real scenarios. Selecting the system parameters as reported in experimental studies for hippocampal pyramidal neurons, i.e., $N_{\max }=10$ [26] and $500 \leq T_{0} \leq 3000$ [29], [30], the capacity of synaptic communication model for information transmission among these neurons is calculated as $C=0.44 \mathrm{bits} / \mathrm{slot}=110 \mathrm{bits} / \mathrm{s}$. These results can be compared 


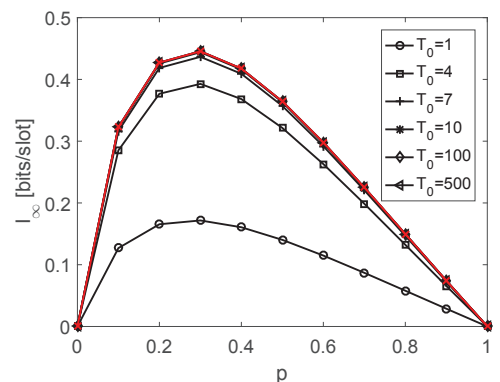

(a) $\lim _{n \rightarrow \infty} \sum_{l=1}^{n} \frac{I\left(S_{l} ; V_{l}\right)}{n}=I_{\infty}$

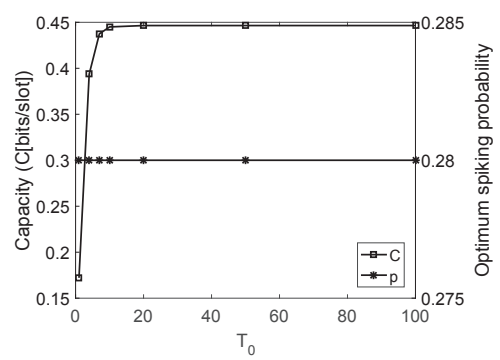

(b) Channel capacity and optimum spiking probability

Fig. 10. Average mutual information of the system, system capacity and capacity-achieving spiking probability in steady state for $N_{\max }=10$ and different values of $T_{0}$.

with the results obtained from the diseased synapses to have an ICT-inspired diagnosis technique for neurodegenerative diseases. Moreover, for designing a molecular communication based nanonetworks, these results can be used to decide about the optimal parameters to achieve maximum capacity.

\section{CONCLUSion}

In this paper, we analyzed a realistic physical reference model as the most basic realization of nanonetworks that utilize neuro-spike communication paradigm from information theoretical perspective. We formulated the mutual information between input and output of a SISO synaptic communication for a single transmission. Since this system has memory, we found the average mutual information over multiple transmissions to evaluate its overall capacity. Moreover, we derived a closed-form expression for the capacity of synaptic transmission and calculated the capacity-achieving spiking probability. Finally, we found the effects of change in various synaptic parameters, such as spiking probability, number of readily releasable vesicles (RRVs) and number of neurotransmitters in a vesicle, on the information capacity of the system.

\section{ACKNOWLEDGMENT}

This work was supported in part by ERC project MINERVA (ERC-2013-CoG \#616922), EU project CIRCLE (EU-H2020FET-Open \#665564), and TUBİTAK graduate scholarship program (BIDEB-2215).

\section{REFERENCES}

[1] I. F. Akyildiz et al., "Nanonetworks: A new communication paradigm," Computer Networks, vol. 52, no. 12, pp. 2260-2279, 2008.
[2] O, B. Akan et al., "Fundamentals of molecular information and communication science," Proc. of the IEEE, to be published, 2016.

[3] T. Nakano et al., "Molecular communication and networking: Opportunities and challenges," IEEE trans. on nanobioscience, vol. 11, no. 2 pp. 135-148, 2012.

[4] E. Balevi and O. B. Akan, "A physical channel model for nanoscale neuro-spike communications," IEEE Trans. Commun., vol. 61, no. 3 , pp. 1178-1187, 2013

[5] H. Ramezani and O. B. Akan, "Synaptic channel model including effects of spike width variation," in Proc. of ACM NANOCOM'15, 2015, p. 11.

[6] D. Malak and O. B. Akan, "A communication theoretical analysis of synaptic multiple-access channel in hippocampal-cortical neurons," IEEE Trans. commun., vol. 61, no. 6, pp. 2457-2467, 2013.

[7] A. Borst and F. E. Theunissen, "Information theory and neural coding." Nature neuroscience, vol. 2, no. 11, 1999.

[8] L. Kostal and R. Kobayashi, "Optimal decoding and information transmission in hodgkin-huxley neurons under metabolic cost constraints," Biosystems, vol. 136, pp. 3-10, 2015.

[9] A. Manwani and C. Koch, "Detecting and estimating signals over noisy and unreliable synapses: information-theoretic analysis," Neural computation, vol. 13, no. 1, pp. 1-33, 2001.

[10] M. Veletić et al., "On the upper bound of the information capacity in neuronal synapses," IEEE Trans Commun., vol. 64, no. 12, pp. 50255036, 2016.

[11] L. Galluccio et al., "Characterization of molecular communication among implantable biomedical neuro-inspired nanodevices," Nano Communication Networks, vol. 4, no. 2, pp. 53-64, 2013

[12] H. Ramezani et al., "Rate region analysis of multi-terminal neuronal nanoscale molecular communication channel," in Proc. of IEEE Nano'17, 2017

[13] V. Matveev and X.-J. Wang, "Implications of all-or-none synaptic transmission and short-term depression beyond vesicle depletion: computational study," J. Neurosci., vol. 20, no. 4, pp. 1575-1588, 2000.

[14] H. Ramezani and O. B. Akan, "Information capacity of vesicle release in neuro-spike communication," IEEE Communications Letters, 2017.

[15] D. Mann and P. Yates, "Neurotransmitter deficits in alzheimer's disease and in other dementing disorders." Human neurobiology, vol. 5, no. 3, pp. 147-158, 1986

[16] M. F. Bear et al., Neuroscience: Exploring the Brain, 3rd ed. Lippincott Williams \& Wilkins, 2007.

[17] J. De La Rocha and N. Parga, "Short-term synaptic depression causes a non-monotonic response to correlated stimuli," J. Neurosci., vol. 25, no. 37, pp. 8416-8431, 2005.

[18] H. Ramezani and O. B. Akan, "Importance of vesicle release stochasticity in neuro-spike communication," in Proc. of IEEE EMBC'17, 2017.

[19] V. N. Murthy and C. F. Stevens, "Reversal of synaptic vesicle docking at central synapses," Nature neuroscience, vol. 2, pp. 503-507, 1999.

[20] T. Khan et al., "Diffusion-based model for synaptic molecular communication channel," IEEE Transactions on NanoBioscience, 2017.

[21] A. Destexhe et al., "kinetic models for synaptic interactions," The Handbook of Brain Theory and Neural Networks, pp. 1126-1130, 2002.

[22] M. Fernandez and S. Williams, "Closed-form expression for the poissonbinomial probability density function," Aerospace and Electronic Systems, IEEE Transactions on, vol. 46, no. 2, pp. 803-817, April 2010.

[23] L. P. Savtchenko and D. A. Rusakov, "The optimal height of the synaptic cleft," Proc Natl Acad Sci U S A, vol. 104, no. 6, pp. 1823-1828, 2007.

[24] T. A. Nielsen et al., "Modulation of glutamate mobility reveals the mechanism underlying slow-rising ampar epses and the diffusion coefficient in the synaptic cleft," Neuron, vol. 42, no. 5, pp. 757-771, 2004.

[25] J. Montes et al., "The influence of synaptic size on ampa receptor activation: A monte carlo model," PloS one, vol. 10, no. 6, p. e0130924 2015

[26] N. Agmon and A. L. Edelstein, "Collective binding properties of receptor arrays." Biophys. J., vol. 72, no. 4, p. 1582, 1997.

[27] L. E. Dobrunz and C. F. Stevens, "Heterogeneity of release probability, facilitation, and depletion at central synapses," Neuron, vol. 18, no. 6, pp. 995-1008, 1997.

[28] T. Schikorski and C. F. Stevens, "Quantitative ultrastructural analysis of hippocampal excitatory synapses," J. Neurosci., vol. 17, no. 15, pp. 5858-5867, 1997

[29] D. A. Rusakov et al., "Shaping the synaptic signal: molecular mobility inside and outside the cleft," Trends in neurosciences, vol. 34, no. 7, pp. 359-369, 2011

[30] F. Ventriglia and V. Di Maio, "A brownian simulation model of glutamate synaptic diffusion in the femtosecond time scale," Biological cybernetics, vol. 83, no. 2, pp. 93-109, 2000. 